\title{
Performance Characterization of Spark Workloads on Shared NUMA Systems
}

\author{
Shuja-ur-Rehman Baig* ${ }^{* \dagger}$, Marcelo Amaral ${ }^{* \ddagger}$, Jordà Polo*, David Carrera*‡ \\ \{shuja.baig, marcelo.amaral, jorda.polo, david.carrera\}@bsc.es \\ ¥Universitat Politècnica de Catalunya (UPC) \\ *Barcelona Supercomputing Center (BSC) \\ †University of the Punjab (PU)
}

\begin{abstract}
As the adoption of Big Data technologies becomes the norm in an increasing number of scenarios, there is also a growing need to optimize them for modern processors. Spark has gained momentum over the last few years among companies looking for high performance solutions that can scale out across different cluster sizes. At the same time, modern processors can be connected to large amounts of physical memory, in the range of up to few terabytes. This opens an enormous range of opportunities for runtimes and applications that aim to improve their performance by leveraging low latencies and high bandwidth provided by RAM. The result is that there are several examples today of applications that have started pushing the in-memory computing paradigm to accelerate tasks. To deliver such a large physical memory capacity, hardware vendors have leveraged Non-Uniform Memory Architectures (NUMA).

This paper explores how Spark-based workloads are impacted by the effects of NUMA-placement decisions, how different Spark configurations result in changes in delivered performance, how the characteristics of the applications can be used to predict workload collocation conflicts, and how to improve performance by collocating workloads in scale-up nodes. We explore several workloads run on top of the IBM Power8 processor, and provide manual strategies that can leverage performance improvements up to $40 \%$ on Spark workloads when using smart processorpinning and workload collocation strategies.
\end{abstract}

Index Terms-Performance, Modeling, Characterization, Memory, NUMA, Spark, Benchmark

\section{INTRODUCTION}

Nowadays, due to the growth in the number of cores in modern processors, parallel systems are built using NonUniform Memory Architecture (NUMA), which has gained wide acceptance in the industry, setting the new standard for building new generation enterprise servers. These processors can be connected to large amounts of physical memory, in the range of up to a couple of terabytes for the time being. This opens an enormous range of opportunities for runtimes and applications that aim to improve their performance by leveraging low latencies and high bandwidth provided by RAM. The result is that today there are several examples of applications that have started pushing the in-memory computing paradigm to accelerate tasks.

To deliver such a large physical memory capacity, sockets in NUMA systems are connected through high performance connections and each socket can have multiple processors with its own memory. A process running on a NUMA system can access the memory of its own node as well remote node where the latency of memory accesses on remote nodes is significantly high compared to local memory accesses [1]. Ideally, memory accesses are kept local in order to avoid this latency and contention on interconnect links. Moreover, the bandwidth of memory accesses to last-level caches and DRAM memory also depends on the access type that is local or remote. From the NUMA perspective, people want to learn whether NUMA topology can meet the challenges of in-memory computing frameworks, and if not, what kinds of optimizations are required.

At the same time, as the adoption of Big Data technologies becomes the norm in many scenarios, there is a growing need to optimize them for modern processors. Spark [2] has gained momentum over the last few years among companies looking for high performance solutions that can scale out across different cluster sizes. To achieve a good performance for in-memory computing frameworks on a NUMA system, there is a need to understand the topology of the interconnect between processor sockets and memory banks. Additionally, while a NUMA architecture can provide very high memory capacity to the applications, it also implies the additional complexity of letting the Operating System take care of many critical decisions with respect to where data is physically stored and where are processes accessing that data placed. This fact may have no impact for many applications that are not memory intensive, whereas memory-bound applications can be seriously impacted by these decisions in terms of performance.

This paper explores how Spark-based in-memory computing workloads are impacted by the effects of NUMA architecture, how different Spark configurations result in changes in delivered performance, how the characteristics of the applications can be used to predict workload collocation conflicts, and how to leverage memory-consumption patterns to smartly co-locate workloads in scale-up nodes. The evaluation also characterizes several workloads running on top of the IBM Power8 processor, and provides strategies that can lead to performance improvements of up to $40 \%$ on Spark workloads when using smart processor-pinning and workload collocation strategies.

In summary, the main contributions of this paper are the following:

- A characterization of three representative memoryintensive Spark workloads across multiple software con- 
figurations on top of a modern NUMA processor (IBM Power 8). The study illustrates how these workloads require a high level of concurrence to achieve full processor utilization when running in isolation, but at the same time they are limited by the processor memory bandwidth when such levels of concurrence is reached, resulting in poor performance and resource underutilization.

- An evaluation of how process and memory binding to NUMA nodes can provide means to improve the performance of memory-intensive Spark workloads by reducing the competition among software threads on high concurrency situations.

- Propose smart workload co-location strategies that leverage process and memory binding to NUMA nodes as a mechanism to improve performance and resource utilization for modern NUMA processors running memoryintensive workloads.

The rest of the paper is organized as follows. Sections II and III provide technological background as well as set the state of the art for the work presented in this paper. Section IV introduces the evaluation methodology used for the experiments. Sections V, VI and VII describe experiments performed to support the conclusions presented in this work. Finally, Section VIII presents the conclusions to the work.

\section{BACKGROUND}

\section{A. Apache Spark}

Apache Spark [2] is a popular open-source platform for large-scale in-memory data processing developed at UC Berkeley. Spark is designed to avoid the file system as much as possible, retaining most data resident in distributed memory across phases in the same job. Spark uses Resilient Distributed Datasets (RDDs) [3] which are immutable collections of objects spread across a cluster and hides the details of distribution and fault-tolerance for a larger collection of items. Spark provides a programming interface based on the two highlevel operations i) transformations ii) actions. Transformations are lazy operations that create new RDDs. Actions launch a computation on RDDs to return a value to the application or write data to the distributed storage system. When a user runs an action on an RDD, Spark first builds a directed acyclic graph (DAG) of stages. Next, it splits the DAG into stages that contain pipelined transformations with dependencies. Further, it divides each stage into tasks. A task is a combination of data and computation. Spark executes all the tasks of a stage before going to next stage. Spark maintains a pool of executor threads which are used to execute the tasks in parallel.

\section{B. IBM Power 8}

We run all the experiments in the IBM Power System 824742L, which is 2-way 12-core IBM Power8 machine with all cores at $3.02 \mathrm{GHz}$ and with each core able to run up to 8 Simultaneous Multi-Threading (SMT) [4]

Each Power8 processor is composed of two memory regions (i.e NUMA node) with 6 cores and their own memory controller and 256GB of RAM. The Power8 processor includes four cache levels, consisting of a store-through L1 data cache, a store-in L2 cache, an eDRAM-based L3 cache with a percore capacity of $64 \mathrm{~KB}, 512 \mathrm{~KB}$, and $8 \mathrm{MB}$, respectively. The fourth cache level has $128 \mathrm{MB}$ and consists of eight external memory chips called Centaur (which is a DDR3 memory).

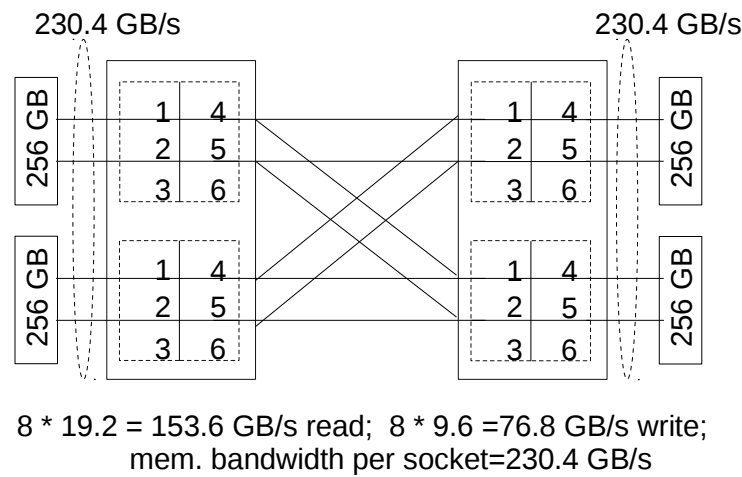

Fig. 1: Power8 NUMA architecture

Because of the main memory is connected to the processor using separate links for reading and write operations, with two links for memory reads and one link for memory writes, the system has asymmetric read and write bandwidth. The connection between the processor with the memory is composed of 8 links, with a link offering $9.6 \mathrm{~GB} / \mathrm{s}$ write and 19.2 $\mathrm{GB} / \mathrm{s}$ read bandwidth. Therefore, the system has in total four NUMA nodes, 192 virtual cores, 1 TB of RAM and a total of $230.4 \mathrm{~GB} / \mathrm{s}$ of sustainable memory bandwidth per socket, as illustrated in Figure 1. For the software stack, the machine is configured with a Ubuntu 16.10, kernel 4.4.0-47-generic, IBM java version 1.8.0 and Apache Spark 1.6.1.

\section{RELATED WORK}

Although there have been several research efforts to investigate and mitigate the impact of NUMA on workload performance, this topic is still gaining traction in the literature in recent years [5], [6], [7], [8], [9], [10] [11], [12], [13]. These works characterize some key sources of workload performance degradation related to NUMA (such as additional latencies and possibly lower memory bandwidth because of remote memory access and contentions on the memory controller, bus connections or cache contention), and propose OS task placement strategies for mitigating remote memory access. But the characterization of Spark workloads on IBM Power8 systems and placement strategies for co-scheduled application is still roughly understood.

For example, [6] has characterized the NUMA performance impact related to remote memory access introduced by the OS when performing task re-scheduling or load balancing. While this work proposed an effective approach to mitigating remote memory access and cache contention, it is not applicationdriven and does not have a holistic view of all applications to define efficient workloads co-scheduling on NUMA systems. In our work, we demonstrate the potential benefits from manual binding strategies when co-scheduling multiple workloads on NUMA systems. 
Another example is the work of [14], where the authors characterized the performance impact of NUMA on graphanalytics applications. They present an approach to minimize remote memory access by using graph-aware data allocation and access strategies. While this work presents an applicationdriven investigation, it lacks the analyze of memory-intensive Spark workloads and workload collocation.

The most related works to our work are the [15] and [16]. In the former, the authors quantify the impact of data locality on NUMA nodes for Spark workloads on Intel Ivy Bridge server. In the later, the authors evaluate the impact of NUMA locality on the performance of in-memory data analytics with Spark on Intel Ivy Bridge server. In both papers, they run benchmarks with two configurations a) Local DRAM b) Remote DRAM. In Local DRAM, they bound Spark process to processor 0 and memory node 0 and in Remote DRAM, they bound the Spark process to processor 0 and memory node 1 . Then, they compare the results to evaluate the performance impact of NUMA. While those works present a detailed performance characterization of Spark workloads on NUMA systems on an Intel Ivy Bridge server, the NUMA performance characterization of IBM Power8 systems is still not understood. Moreover, their work does not present the NUMA impact of optimally binding the workloads versus leaving the OS allocating the resources. Also, they do not evaluate the performance benefits of performing manual binding for coscheduled Spark workloads as we present in this paper.

\section{Methodology}

This section describes how the study on the impact of NUMA topology on in-memory data analytics workloads has been performed, as well as the rationale behind the experiments evaluated in the following sections.

\section{A. Workloads}

The experiments presented in this paper are based on SparkBench [17], which is a benchmark suite developed by IBM and widely tested in Power8 systems. From the range of available workloads provided by the benchmark, Support Vector Machines (SVM), PageRank, and Spark SQL RDDRelation have been selected for the evaluation. These workloads are wellknown in the literature, and combine different characteristics to cover a large range of possible configurations. Dataset size for SVM, SQL and PageRank is 47, 24, and $17 \mathrm{~GB}$ and number of partitions are 376, 192 and 136 respectively for all experiments.

\section{B. Experimental Setup}

Since the goal of this paper is to evaluate the performance of Spark workloads on NUMA hardware, all the experiments are conducted in a single machine; the characteristics of the machine's architecture are described in Section II-B. For simplicity, Spark is configured in the standalone mode [18], To control the number of cores, memory, and the number of executors of each worker, the parameters SPARK_WORKER_CORES, SPARK_WORKER_MEMORY, and

\begin{tabular}{ll}
\hline parameter & value \\
\hline spark.serializer & org.apache.spark.serializer.KryoSerializer \\
\hline spark.rdd.compress & FALSE \\
\hline spark.io.compression.codec & lzf \\
\hline storage level & MEMORY_AND_DISK \\
\hline spark.driver.maxResultSize & $2 \mathrm{gb}$ \\
\hline spark.driver.memory & $5 \mathrm{gb}$ \\
\hline spark.kryoserializer.buffer.max & $512 \mathrm{~m}$ \\
\hline
\end{tabular}

TABLE I: Spark configuration parameters

SPARK_EXECUTOR_MEMORY [18] are used, respectively. All the other parameters and values used to configure Spark, during the experiments execution described later in this paper, are summarized in Table I.

Hardware counters have been used to collect most realtime information from experimental executions, using the perfmon2 [19] library. Memory bandwidth is calculated based on the formula defined in [20]. For CPU usage, memory usage, and context switches, the vmstat tool has been used. To collect information about NUMA memory access, the numastat is used. Finally, the numact 1 command has been used to bind a workload in a set of CPUs or in memory regions (e.g. in a NUMA node); the $\mathrm{nB}$ nomenclature is used to describe different binding configurations, where $\mathrm{n}$ is the number of assigned NUMA nodes, e.g. $1 B$ for workloads bound to 1 NUMA node, $2 B$ for workloads bound to 2 NUMA nodes, etc.

\section{EXPERIMENT 1: WORKLOAD CHARACTERIZATION}

This experiment consists of a performance characterization of Spark workloads, changing the configuration parameters of Spark itself and observing the impact of different configurations in terms of completion time and resource consumption. The goal is to find which configurations lead to optimal performance, e.g. which number of cores per Spark worker, and/or the number of workers per application. Since this experiment aims at defining the software configuration, there is no other kind of hardware tuning involved; the OS performs its default resource allocation decisions. More specifically, this experiment analyzes the effect of the software configuration in the resource usage intensiveness and possible speedups for the workloads described in Section IV-A.

As described in Section II-B, the machine used for the experiments has 4 NUMA nodes, 192 virtual cores, and 1TB of main memory. Thus, this experiment varies the number of cores, workers, and memory up to the total amount of resources (cores and memory) available in this machine. Table II describes all combinations of the amount of resources allocated to all workloads in this experiment. The amount of memory ranges from 5 up to $250 \mathrm{~GB}$ per worker, the number workers vary from 4 up to 192 workers. Depending on the number of workers, the number of virtual cores ranges from 1 up to 192. If the total number of workers is 192, each one will have only one virtual core. Note that, by creating this matrix of experiments, we want to see at which configuration, the operating system produce optimal results for each workload type in terms of completion time. We assign memory to Spark 


\begin{tabular}{|c|c|c|c|c|c|c|c|c|c|c|c|c|}
\hline \multirow{2}{*}{$\begin{array}{l}\mathbf{w} \\
\text { e } \\
\mathbf{m}\end{array}$} & \multirow{2}{*}{$\begin{array}{l}\mathbf{t} \\
\mathbf{m} \\
\mathbf{a}\end{array}$} & \multirow{2}{*}{$\begin{array}{l}\mathbf{t} \\
\mathbf{s} \\
\mathbf{w}\end{array}$} & \multicolumn{10}{|c|}{ core per worker } \\
\hline & & & 1 & 2 & 3 & 4 & 6 & 8 & 12 & 16 & 24 & 48 \\
\hline 250 & 1000 & 4 & 4 & 8 & 12 & 16 & 24 & 32 & 48 & 64 & 96 & 192 \\
\hline 125 & 1000 & 8 & 8 & 16 & 24 & 32 & 48 & 64 & 96 & 128 & 192 & \\
\hline 83 & 996 & 12 & 12 & 24 & 36 & 48 & 72 & 96 & 144 & 192 & & \\
\hline 62 & 992 & 16 & 16 & 32 & 48 & 64 & 96 & 128 & 192 & & & \\
\hline 41 & 984 & 24 & 24 & 48 & 72 & 96 & 144 & 192 & & & & \\
\hline 31 & 992 & 32 & 32 & 64 & 96 & 128 & 192 & & & & & \\
\hline 20 & 960 & 48 & 48 & 96 & 144 & 192 & & & & & & \\
\hline 15 & 960 & 64 & 64 & 128 & 192 & & & & & & & \\
\hline 10 & 960 & 96 & 96 & 192 & & & & & & & & \\
\hline 5 & 960 & 192 & 192 & & & & & & & & & \\
\hline
\end{tabular}

TABLE II: Experiment 1: Evaluated software configurations (wem is worker and executor memory; tma is total memory allocated; and $t s w$ is total Spark workers)

\begin{tabular}{|l|l|l|l|l|l|l|}
\hline workload & $\begin{array}{l}\text { total } \\
\text { workers }\end{array}$ & $\begin{array}{l}\text { core } \\
\text { per } \\
\text { worker }\end{array}$ & $\begin{array}{l}\text { total } \\
\text { cores } \\
\text { allocated }\end{array}$ & $\begin{array}{l}\text { worker } \\
\text { executor } \\
\text { memory }\end{array}$ & $\begin{array}{l}\text { total } \\
\text { memory } \\
\text { allocated }\end{array}$ & $\begin{array}{l}\text { Execution } \\
\text { Time (sec) }\end{array}$ \\
\hline SVM & 24 & 8 & 192 & 41 & 984 & 323.71 \\
\hline SQL & 4 & 12 & 48 & 250 & 1000 & 206.82 \\
\hline PageRank & 12 & 8 & 96 & 83 & 996 & $748.08:$ \\
\hline
\end{tabular}

TABLE III: Experiment 1: Best configuration when optimizing for completion time

worker and executor by dividing 1000 by a total number of workers in the experiment and take the integral part only; thus, the amount of memory ranges from $960 \mathrm{~GB}$ to $1000 \mathrm{~GB}$. Some amount of memory is intentionally left for the OS and other processes (e.g spark driver, master) to avoid the slowdown effects not related to NUMA.

In Spark, a software configuration defines the number of workers, the number virtual cores and the amount of memory per worker that is assigned to a specific workload. These software resources need to match the hardware configuration of the node used to run the workloads. But not all applications can take advantage of an increasing amount of resources and therefore it is not always the case that one single software configuration optimizes the performance of a Spark Workload for a given hardware setup.

Table III summarizes the optimal configurations that found for the three workloads considered in this experiment. As it can be seen, every workload achieves maximum performance using a different software configuration, being SVM the application that can take advantage of more threads in parallel, followed by PageRank and finally SQL. It is remarkable that even configurations with a similar number of cores allocated tend to deliver different performance for similar configurations of number of workers and number of cores per worker. For instance, SQL works best with fewer workers and more cores per worker and SVM gets the best performance when more workers and fewer cores per worker are assigned. This is due to SQL is more impacted because of thread locks and cache contention than the SVM. Hence, SQL benefits from fewer threads competing for resources. Additionally, because the JVM includes additional overheads (e.g. garbage collection), more layers for resource management and memory indexing, it is not beneficial to have several workers with only one virtual core.

To explain the root cause of the performance delivered by the different configurations, Tables IV , V and VI also show the executions times in seconds obtained for SVM , SQL, and PageRank respectively when using all combinations of software configurations, but in this case, we color each configuration according to the relative performance delivered compared to the optimal configuration found for that particular workload. Based on this property we classify configurations in different groups:

- Within 10\% of optimal: configurations for which completion time is very close to the best execution time observed for that particular workload.

- Low CPU Usage: configurations for which CPU usage is clearly below the observed CPU usage for the workload. These configurations use a too low number of cores or workers that are not enough to fully utilize the available compute resources and produce optimal results.

- High CPI and Context Switches: executions where cycles per instruction (CPI) and context switches are greater than the observed values for the optimal configuration. This is due to more executors which execute more threads to process the tasks. Moreover, executors need to communicate with each other and also with drivers. Remote memory access also impacts the CPI since it requires more CPU cycles to be performed than local access. This leads to increase in communication overhead. So in result, we see an increase in context switches and CPI.

- High L3 misses: configurations where L3 cache misses are greater than in the optimal configuration. This group is only defined for SVM as it is the only workload for which this behavior was observed.

- Low Memory Bandwidth: configurations where memory bandwidth usage is less than the observed value for the optimal configuration.

- Require more investigation: configurations where values of metrics are within the range of optimal region but the completion time is outside of $10 \%$. The experiments in this region require further investigation and it could not be determined so far the reason for the performance differences with the optimal configuration.

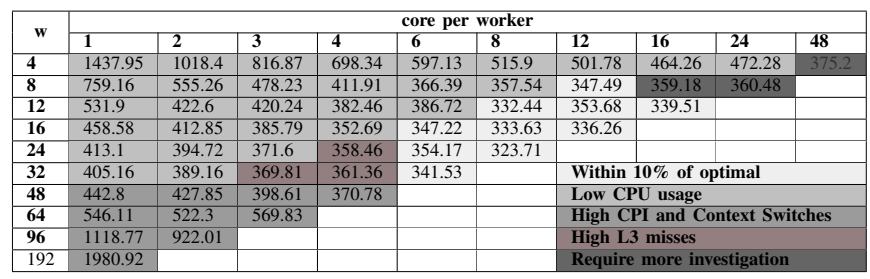

TABLE IV: SVM completion time (seconds) groups

The second objective of this experiment was to characterize the CPU, Memory Footprint and Memory Bandwidth demands of each one of the workloads of study. For this purpose, we monitored the execution of the workloads when the optimal software configuration was in use and plotted the average resource consumption in Figure 2. As it can be seen, results show that memory usage is $457.7 \mathrm{~GB}, 364.3 \mathrm{~GB}$ and 329.4 GB for SVM, SQL and PageRank respectively and shows the 


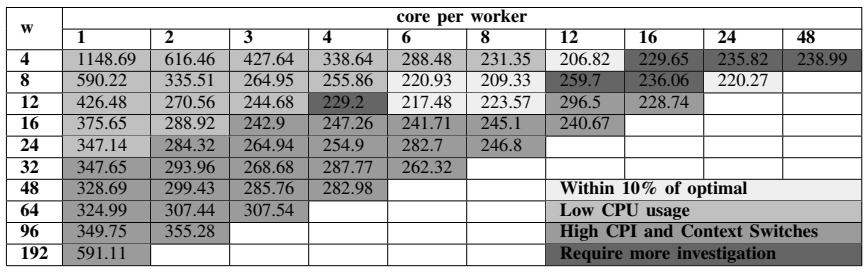

TABLE V: SQL completion time (seconds) groups

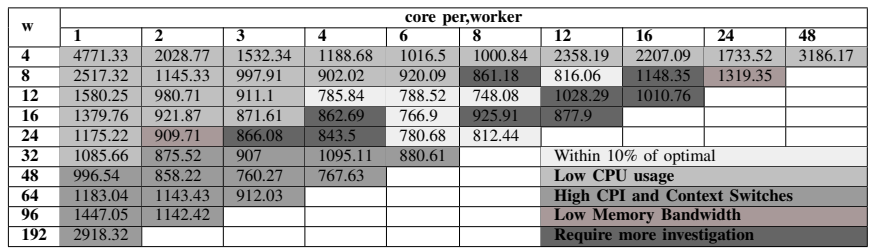

TABLE VI: PageRank completion time (seconds) groups

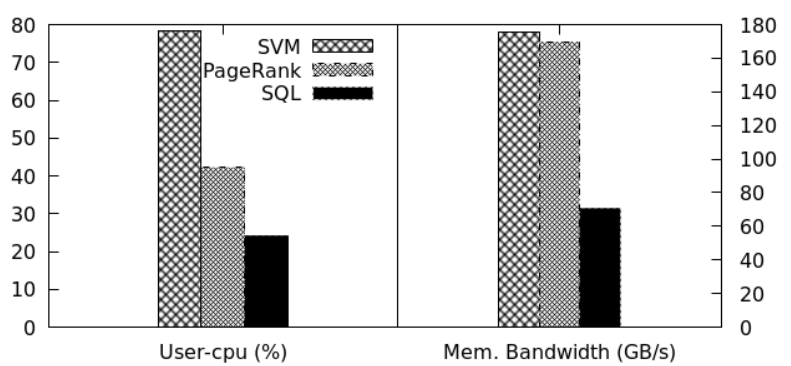

Fig. 2: Experiment 1: CPU Usage (percentage) and Memory Bandwidth (GB/s) for optimal configuration

average usage of user CPU time and memory bandwidth for these workloads when the optimal software configuration is in use. As it can be observed, SVM is constrained by the high CPU usage, reaching around $80 \%$ for the user CPU time only, that when added to the system and wait CPU times tops to about $100 \%$ CPU usage, which is the actual performance bottleneck.

SQL is a more interesting case because CPU and Memory Bandwidth usage are really low for the fastest configuration, and no other resource is apparently acting as a bottleneck. In practice, what is avoiding the total CPU usage to go higher is the fact that the number of threads that are spawn in this configuration (only 48) is well below the number of hardware threads offered by the system. Therefore, only a third of the hardware threads are in use and that is why the average CPU utilization is shown to be low: several hardware threads are idle. Intuition would say that increasing the number of threads would increase the performance, but in practice what is observed in the logs of other experiments is that as soon as the number of threads goes higher the memory bandwidth dramatically increases, quickly becoming the bottleneck at many stages during the execution. The bottom line is that the OS is not able to correctly manage the threads for this workload, creating memory access patterns that saturate the memory links of the $\mathrm{P} 8$ processor.

Finally, PageRank is in the same situation: the optimal configuration involves 96 software threads only, while the system offers 192 hardware threads. In practice, what it means is that the reported CPU utilization is low. Intuition again would point in the direction of increasing the number of software threads, but when that direction is taken, logs show that the additional software threads start competing for memory bandwidth because they exhibit worse memory access patterns, and saturate the memory links.

In summary, this experiment has shown two cases in which not all hardware threads could be exploited because in that case the memory access patterns across NUMA nodes were hitting a memory bandwidth bottleneck. This is an interesting result because opens a door to smart workload collocation strategies that will be explored in the following experiments.

\section{EXPERIMENT 2: BINDING TO NUMA NODES}

Allocating more NUMA nodes to a workload has the potential to increase resources (such as memory bandwidth, $\mathrm{CPU}$, and memory) and possibly lower cache contention (due to the availability of additional cores and cache), but it can also involve a trade-off: using remote memory accesses and dealing with bus contention, which can lead to slowdowns in some scenarios. Binding, in this context, means Spark processes (master and workers) will only have access to the resources (cores and memory) of a particular set of NUMA nodes. While the previous experiment selected the optimal software configuration without binding, allowing the operating system to make all decisions, this experiment selects the optimal software configuration when binding all 4 nodes (4B). Results are also compared to the previous experiment so as to evaluate the impact of binding.

In the previous experiment, the OS was responsible for allocating all the resources, in this experiment we enforce decisions to manually bind the workloads across the NUMA nodes. The main motivation of performing the manual binding is to mitigate the limitations defined in the previous experiments. Manually binding the workloads can exploit better load balancing, minimize thread migrations and remote memory access. In order to verify those assumptions, we evaluate the completion time of all workloads for different binding configurations and compare with non-binding approaches (the default allocation from OS). As explained in Section VI, the workloads are bound in one NUMA node up to $4(1 \mathrm{~B}, 2 \mathrm{~B}$, 3B, 4B). The results of the optimal software configuration considering the different number of NUMA nodes is shown in Table VII. It also shows the comparison of the manual binding with four NUMA nodes versus the default OS resource allocation, labeled as NB.

The optimal configurations are 24 cores per worker and 1 worker per node for SQL, SVM, and PageRank when we bound workloads to one NUMA node (1B). In case of $2 \mathrm{~B}$, the optimal configurations are 8 cores per worker and 3 workers per node for SQL, 6 cores per worker and 6 workers per node for SVM and 4 cores per worker and 6 workers per node for PageRank. Similarly, in case of 3B, the optimal configurations are 8 cores per worker and 2 workers per node for SQL, 6 cores 


\begin{tabular}{llll}
\hline $\mathbf{n}(\mathbf{x})$ to $n(y)$ & SVM & SQL & PageRanK \\
\hline $1 \mathrm{~B} \rightarrow 2 \mathrm{~B}$ & $1.44 \mathrm{x}$ & $1.73 \mathrm{x}$ & $1.79 \mathrm{x}$ \\
\hline $1 \mathrm{~B} \rightarrow 4 \mathrm{~B}$ & $2.07 \mathrm{x}$ & $2.63 \mathrm{x}$ & $2.64 \mathrm{x}$ \\
\hline $2 \mathrm{~B} \rightarrow 4 \mathrm{~B}$ & $1.44 \mathrm{x}$ & $1.52 \mathrm{x}$ & $1.47 \mathrm{x}$ \\
\hline $3 \mathrm{~B} \rightarrow 4 \mathrm{~B}$ & $1.1 \mathrm{x}$ & $1.11 \mathrm{x}$ & $1.13 \mathrm{x}$ \\
\hline $4 \mathrm{NB} \rightarrow 4 \mathrm{~B}$ & $1.15 \mathrm{x}$ & $1.07 \mathrm{x}$ & $1.25 \mathrm{x}$ \\
\hline
\end{tabular}

TABLE VII: Experiment 2: Speedup (B=Node with binding, NB= Node without binding)

per worker and 4 workers per node for SVM and 3 cores per worker and 6 workers per node for PageRank. The optimal configurations in case of $4 \mathrm{~B}$ are 12 cores per worker and 1 worker per node for SQL, 8 cores per worker and 3 workers per node for SVM and 6 cores per worker and 3 workers per node for PageRank.

The results of this experiment, as summarized in Table VII, show a significant speedup when comparing manual binding versus the OS allocating the resources, but not for all workloads. In the case of SVM, SQL and PageRank, the speedups are $\mathrm{x} 1.15, \mathrm{x} 1.07$ and $\mathrm{x} 1.25$, respectively. This is related to what was discussed in the previous experiment, the main bottleneck is related to resource contention, most especially the memory bandwidth. Because of manual binding minimizes remote memory access and local memory access has higher memory bandwidth and lower latency, some workload benefit from that. However, this improvement impacts different each workload what depends on the workload memory access pattern.

The results of this experiment also show that how applications scale with more NUMA nodes. Considering following formula ( current node(s) + new node(s) / current node(s)), the theoretical speedup of allocating one new NUMA node to application with one current node would lead to a speedup of $2 \mathrm{x}$. The results actually show different speedups for all workloads. PageRank is the workload that scales better, with $1.79 \mathrm{x}$ from one (1B) to two (2B) NUMA nodes. In the case of SVM, speedup for $1 \mathrm{~B}-2 \mathrm{~B}$ is only $1.44 \mathrm{x}$. SQL is in between them with a $1.73 \mathrm{x}$ speedup for $1 \mathrm{~B}-2 \mathrm{~B}$. In the case of increasing from 3 to 4 NUMA nodes (3B to $4 B$ ), the theoretical speedup would be $1.33 \mathrm{x}$ and results show the speedup in range of $1.1 \mathrm{x}$ to $1.13 \mathrm{x}$.

\section{EXPERIMENT 3: WORKLOAD CO-SCHEDULING}

This final experiment explores the benefits of workload colocation and process binding (cores and memory) as a mechanism to improve system throughput and increase resource utilization. Workload co-location is well-known to possibly slow down interference-sensitive applications, however, the impact of NUMA co-scheduled Spark workloads are still not completely understood. This experiment, therefore, evaluates the performance impact on workloads when sharing the same machine, that is when workloads are co-located. In order to do so, a defined set of workload combinations among the four NUMA nodes is provided. This experiment aims at evaluating the trade-offs between sharing NUMA nodes without binding (with the OS using simple resource allocation policies), versus binding the workloads to isolate their interference from each other.

\begin{tabular}{|l|l|l|l|l|l|l|l|l|}
\hline & & & w1 & w2 & $\mathbf{w 1}$ & $\mathbf{w 2}$ & $\mathbf{w 1}$ & $\mathbf{w 2}$ \\
\hline n-n & w1 & w2 & w/n & w/n & $\mathbf{c / w}$ & $\mathbf{c / w}$ & $\mathbf{w + e}$ & $\mathbf{w + e}$ \\
\hline $2-2$ & SQL & SVM & 3 & 6 & 8 & 6 & 83 & 41 \\
\hline $2-2$ & SQL & PR & 3 & 6 & 8 & 4 & 83 & 41 \\
\hline $2-2$ & SVM & PR & 6 & 6 & 6 & 4 & 41 & 41 \\
\hline $1-3$ & SQL & SVM & 1 & 4 & 24 & 6 & 250 & 62 \\
\hline $1-3$ & SQL & PR & 1 & 6 & 24 & 3 & 250 & 41 \\
\hline $1-3$ & SVM & PR & 1 & 6 & 24 & 3 & 250 & 41 \\
\hline $3-1$ & SQL & SVM & 2 & 1 & 8 & 24 & 125 & 250 \\
\hline $3-1$ & SQL & PR & 2 & 1 & 8 & 24 & 125 & 250 \\
\hline $3-1$ & SVM & PR & 4 & 1 & 6 & 24 & 62 & 250 \\
\hline
\end{tabular}

TABLE VIII: Configurations for different co-scheduling workload combinations; (n-n=NUMA node combination; w1=workload-1; w2=workload-2; w/n=worker per node; $\mathrm{c} / \mathrm{w}=$ =core per worker; $\mathrm{w}+\mathrm{e}=$ worker and executor memory; $P R=$ PageRank)

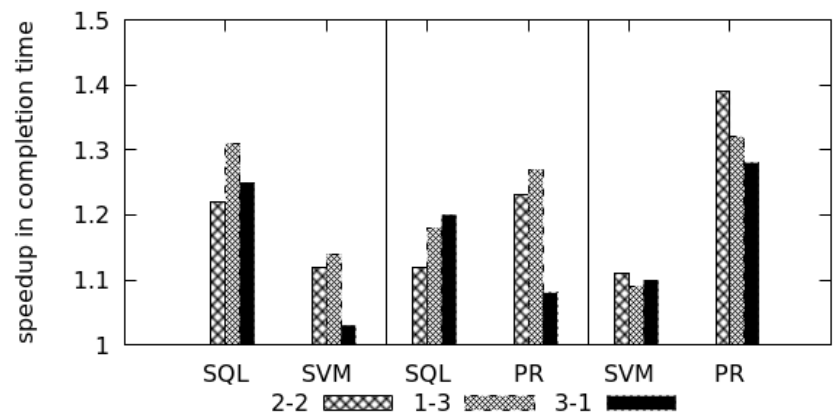

Fig. 3: Experiment 3: Completion time speedup (60 minutes interval; binding vs non-binding(OS default allocation))

The baseline for this experiment is based on the optimal configurations from Experiment 2. Table VIII describes all the workload co-location scenarios along with their software configurations. For all configurations evaluated in this experiment, only two workloads run at the same time. First, the workloads are equally spread to the four NUMA nodes. That is, each workload is allocated to two NUMA nodes, labeled as 2B-2B. Secondly, uneven configurations are evaluated, such as $1 \mathrm{~B}-3 \mathrm{~B}$ and $3 \mathrm{~B}-1 \mathrm{~B}$, meaning one of the workloads is bound to 1 NUMA node and the other one to 3 NUMA nodes. Note that all configurations are executed with binding on NUMA nodes according to the description in Table VIII, but also without binding where the OS allocates all shared resources.

For evaluation purposes, more than one workload is exe-

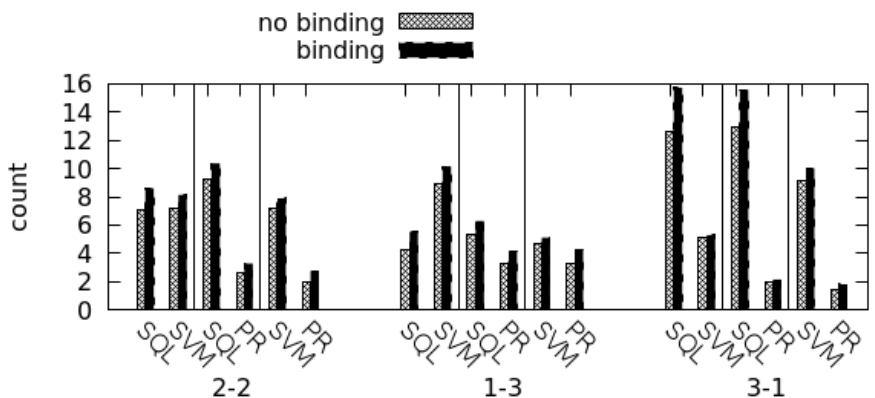

Fig. 4: Experiment 3: Number of executions (60 minutes interval) 
cuted at a time in the system under test. As each workload exhibits different job duration characteristics, we evaluate the effects of co-location on a continuous workload environment. In particular, for any pair of workloads that is evaluated, we run both of them in a continuous loop of 90 minutes, from which the first 15 minutes are taken as a warm-up period and the final 15 minutes as a cool-down process. The central 60 minutes of the experiment are used to measure the performance of the system. During that period we collect system telemetry as well as account for how many loops every workload managed to perform.

The first configuration evaluated makes an even distribution of the NUMA nodes across the two workloads under test. We label this test $2 B-2 B$ (2 NUMA nodes with process binding). Each partition runs a different workload. Each workload is configured with the optimal $2 \mathrm{~B}$ configuration found in the previous experiment. We repeat the process with the $1 B-3 B$ configurations (1B for 1 NUMA node with binding, and 3B for 3 NUMA nodes with binding), in which one workload gets assigned one NUMA node while the other gets allocated the other three nodes.

As it was mentioned before, to provide a baseline for these experiments we also run the same workload co-location experiments, maintaining in every case the software configuration, but in this case without performing any process binding operation and therefore allowing the Operating System to freely schedule processes, threads and memory pages across NUMA nodes. In all cases we executed all combinations of SQL-SVM, SQL-PageRank, SVM-PageRank.

Figure 3 shows the speedup in execution time when binding over non-binding configurations. As it can be observed, in all cases the process binding configurations outperformed the non-binding setups, providing a significant improvement that in some cases reached a 1.39X factor. Figure 4 shows the number of executions during the 60 minute interval for all workloads, and as it can be observed, binding always outperforms non-binding. To explain the reason for this improvement, we looked at different system metrics.

In this section, we include the results for the number of CPU Context Switches and the amount of remote memory accessed per workload, as summarized in Figures 5 and 6 respectively.

We found that there is an increase of $36-43 \%$ in context switches when experiments are executed without binding. When processes are not bound to specific NUMA nodes, the OS is in charge of all placement decisions, which includes reactive migrations to balance the load. In a case of remote memory access, there is an increase of $80-91.93 \%$ when the same experiments are executed without binding. So in conclusion, we show that memory binding reduces the amount of remote memory access and this leads to less interconnect traffic (because of less memory transfer and cache-coherence) and contention on inter-links for Spark workloads. Moreover, CPU binding reduces the number of context switches, which also helps improve completion time for experiments.

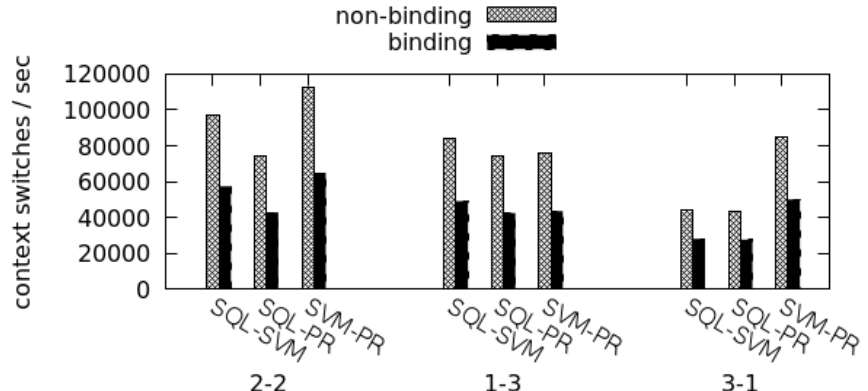

Fig. 5: Experiment 3: Context switches per second (60 minutes interval)

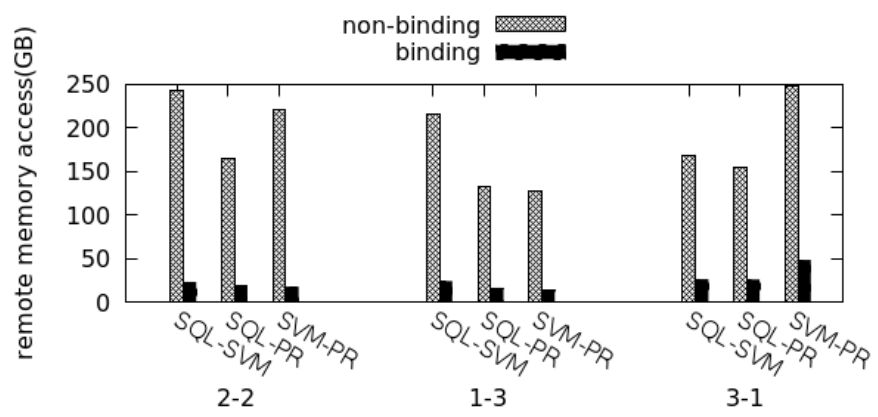

Fig. 6: Experiment 3: Amount of remote memory access in GB (60 minutes interval)

\section{CONCLUSIONS}

In-memory computing is becoming one of the most popular approaches for real-time big data processing as data sets grow and more memory capacity is made available to popular runtimes such as Spark. To deliver large physical memory capacity, modern processors feature Non-Uniform Memory Architectures (NUMA). In NUMA systems, multiple sockets are connected through high-performance connections. Each socket can have multiple processors with its own memory. A process running in a NUMA system can access the memory of its own node as well the remote node, where the latency of memory accesses is higher. It is thus important to understand how the hardware topology of a particular NUMA architecture affects the performance of in-memory computing applications. This paper analyzed the behavior of different memory-intensive Spark workloads on the IBM Power 8 NUMA processor.

Large sets of experiments were executed to evaluate several Spark workloads, and the results demonstrated that workload colocation is a smart strategy to improve resource utilization for memory-intensive workloads placed in modern NUMA processors. This conclusion is supported by the fact that the experiments showed that different kinds of Spark workloads require different software configurations to produce optimal results and that optimal configurations are commonly unable to use all available hardware resources. Highly concurrent configurations produce undesired memory access patterns across NUMA nodes that push to the limit the existing memory 
bandwidth, making co-scheduling a good choice.

Additionally, the experiments provided insight on the existing trade-off between sharing NUMA nodes and isolating workloads; optimal configurations when binding to a different number of NUMA nodes change significantly depending on the workload and the number of nodes used. There is one constant though: when executing a workload in a single NUMA node, using a single Spark worker produces the optimal results for all workloads.

The obtained results show that binding spark processes to particular NUMA nodes can speed up the completion time of co-located workloads up to $1.39 \mathrm{x}$ at maximum due to less interconnect traffic, less remote memory access, and less context switches and CPI.

\section{ACKNOWLEDGMENTS}

This work is partially supported by the European Research Council (ERC) under the EU Horizon 2020 programme (GA 639595), the Spanish Ministry of Economy, Industry and Competitiveness (TIN2015-65316-P) and the Generalitat de Catalunya (2014-SGR-1051).

\section{REFERENCES}

[1] S. Blagodurov, S. Zhuravlev, M. Dashti, and A. Fedorova, "A case for numa-aware contention management on multicore systems," in Proceedings of the 2011 USENIX Conference on USENIX Annual Technical Conference, ser. USENIXATC'11. Berkeley, CA, USA: USENIX Association, 2011, pp. 1-1. [Online]. Available: http://dl.acm.org/citation.cfm?id=2002181.2002182

[2] M. Zaharia, M. Chowdhury, M. J. Franklin, S. Shenker, and I. Stoica, "Spark: cluster computing with working sets."

[3] M. Zaharia, M. Chowdhury, T. Das, A. Dave, J. Ma, M. McCauley, M. J. Franklin, S. Shenker, and I. Stoica, "Resilient distributed datasets: A fault-tolerant abstraction for in-memory cluster computing," in Proceedings of the 9th USENIX conference on Networked Systems Design and Implementation. USENIX Association, 2012, pp. 2-2.

[4] B. Sinharoy, J. Van Norstrand, R. J. Eickemeyer, H. Q. Le, J. Leenstra, D. Q. Nguyen, B. Konigsburg, K. Ward, M. Brown, J. E. Moreira et al., "Ibm power8 processor core microarchitecture," IBM Journal of Research and Development, vol. 59, no. 1, pp. 2-1, 2015.

[5] R. P. LaRowe Jr, C. S. Ellis, and L. S. Kaplan, "The robustness of numa memory management," in ACM SIGOPS Operating Systems Review, vol. 25, no. 5. ACM, 1991, pp. 137-151.

[6] S. Blagodurov, S. Zhuravlev, and A. Fedorova, "Contention-aware scheduling on multicore systems," ACM Transactions on Computer Systems (TOCS), vol. 28, no. 4, p. 8, 2010.

[7] Z. Majo and T. R. Gross, "Memory management in numa multicore systems: trapped between cache contention and interconnect overhead," in ACM SIGPLAN Notices, vol. 46, no. 11. ACM, 2011, pp. 11-20.

[8] C. Lameter, "Numa (non-uniform memory access): An overview," Queue, vol. 11, no. 7, p. 40, 2013.

[9] J. Ahn, C. Kim, J. Han, Y.-r. Choi, and J. Huh, "Dynamic virtual machine scheduling in clouds for architectural shared resources." in HotCloud, 2012.

[10] I. Psaroudakis, T. Scheuer, N. May, A. Sellami, and A. Ailamaki, "Adaptive numa-aware data placement and task scheduling for analytical workloads in main-memory column-stores," Proceedings of the $V L D B$ Endowment, vol. 10, no. 2, pp. 37-48, 2016.

[11] A. Drebes, A. Pop, K. Heydemann, N. Drach, and A. Cohen, "Numaaware scheduling and memory allocation for data-flow task-parallel applications," in Proceedings of the 21st ACM SIGPLAN Symposium on Principles and Practice of Parallel Programming. ACM, 2016, p. 44.

[12] A. Drebes, A. Pop, K. Heydemann, A. Cohen, and N. Drach, "Scalable task parallelism for numa: A uniform abstraction for coordinated scheduling and memory management," in Parallel Architecture and Compilation Techniques (PACT), 2016 International Conference on. IEEE, 2016, pp. 125-137.
[13] I. Psaroudakis, T. Scheuer, N. May, A. Sellami, and A. Ailamaki, "Scaling up concurrent main-memory column-store scans: Towards adaptive numa-aware data and task placement," Proceedings of the VLDB Endowment, vol. 8, no. 12, pp. 1442-1453, 2015.

[14] K. Zhang, R. Chen, and H. Chen, "Numa-aware graph-structured analytics," in ACM SIGPLAN Notices, vol. 50, no. 8. ACM, 2015, pp. 183-193.

[15] A. J. Awan, M. Brorsson, V. Vlassov, and E. Ayguade, "Architectural impact on performance of in-memory data analytics: Apache spark case study," arXiv preprint arXiv:1604.08484, 2016.

[16] A. J. Awan, V. Vlassov, M. Brorsson, and E. Ayguade, "Node architecture implications for in-memory data analytics on scale-in clusters," in Proceedings of the 3rd IEEE/ACM International Conference on Big Data Computing, Applications and Technologies. ACM, 2016, pp. 237-246.

[17] M. Li, J. Tan, Y. Wang, L. Zhang, and V. Salapura, "Sparkbench: A comprehensive benchmarking suite for in memory data analytic platform spark," in Proceedings of the 12th ACM International Conference on Computing Frontiers, ser. CF '15. New York, NY, USA: ACM, 2015, pp. 53:1-53:8. [Online]. Available: http: //doi.acm.org/10.1145/2742854.2747283

[18] "Apache spark standalone mode." [Online]. Available: https://spark. apache.org/docs/1.6.1/spark-standalone.html

[19] "Perfmon2." [Online]. Available: http://perfmon2.sourceforge.net/

[20] T. Ewart, S. Yates, F. Cremonesi, P. Kumbhar, F. Schürmann, and F. Delalondre, "Performance evaluation of the ibm power8 architecture to support computational neuroscientific application using morphologically detailed neurons," in Proceedings of the 6th International Workshop on Performance Modeling, Benchmarking, and Simulation of High Performance Computing Systems, ser. PMBS '15. New York, NY, USA: ACM, 2015, pp. 1:1-1:11. 